\title{
Reply to Comments on "Structural, Optical, and Electrical Properties of Zn-Doped CdO Thin Films Fabricated by a Simplified Spray Pyrolysis Technique" by K. Usharani and A.R. Balu published in Acta Metall. Sin. (Engl. Lett.) 28(1), 64-71 (2015)
}

\author{
A. R. Balu ${ }^{1}$
}

Received: 15 April 2018/Published online: 21 June 2018

(c) The Chinese Society for Metals and Springer-Verlag GmbH Germany, part of Springer Nature 2018

Following are the comments for the queries raised by Prof. Pawel E. Tomaszewski on our published paper entitled "Structural, Optical, and Electrical Properties of Zn-Doped CdO Thin Films Fabricated by a Simplified Spray Pyrolysis Technique" by K. Usharani and A.R. Balu published in Acta Metall. Sin. (Engl. Lett.) 28(1), 64-71 (2015):

1. The formula for micro-strain evaluation, (3), is wrong. It should be " $\cot \theta$ " instead " $\cos \theta "(\cot \theta=\cos \theta / \sin \theta)$. Unfortunately, this error is rather popular in other papers. This is a formula by Wilson for the broadening of diffraction profile due to micro-strain within the sample. As a result, all data on the micro-strain presented in Table 2 are wrong.

- Yes, I agree that strain must be calculated using the relation, $\varepsilon=\frac{\beta \cot \theta}{4}$ and not $\frac{\beta \cos \theta}{4}$. The strain values in our manuscript are calculated only using $\cot \theta$, and the values given in Table 2 are correct. Unfortunately, the formula given in the manuscript is typed incorrectly. For your verification and to prove the correctness of the values given in Table 2, I submit here the XRD data values what we got for the (1 1 1) plane from which the calculations has been done.

Available online at http://link.springer.com/journal/40195

A. R. Balu

arbalu757@gmail.com

$1 \quad$ PG and Research Department of Physics, AVVM Sri Pushpam College, 757 MIG Colony, New Housing Unit, Thanjavur, Tamilnadu 613 005, India

\begin{tabular}{llll}
\hline $\begin{array}{l}\text { Zn doping } \\
\text { concentration (at\%) }\end{array}$ & $2 \theta\left(^{\circ}\right)$ & $\begin{array}{l}\text { FWHM, } \beta \\
\text { (radian) }\end{array}$ & $\begin{array}{l}\text { Strain, } \\
\varepsilon=\frac{\beta \cot \theta}{4} \times 10^{-3}\end{array}$ \\
\hline 0 & 33.007 & 0.23 & 3.387 \\
2 & 33.097 & 0.29 & 4.259 \\
4 & 33.076 & 0.27 & 3.968 \\
6 & 33.097 & 0.227 & 3.330 \\
8 & 33.105 & 0.24 & 3.523 \\
\hline
\end{tabular}

2. The X-ray diffraction peak of the highest intensity does not point out on the preferred orientation in the corresponding direction. In most of cases, its intensity is only related to the crystal structure of studied crystal, not anymore. This is the case of $\mathrm{CdO}$ (Fig. 1) where the intensities of Bragg peaks agree well with the powder diffraction pattern of crystal. That is why all cited papers have the same result. Thus, there are no preferential orientation along [111] direction!

- The preferential orientation factor $f(h k l)$ value for a particular plane is determined by evaluating the fraction of the intensity of that particular plane over the sum of the intensities of all the other peaks observed in the XRD pattern. The preferential orientation is along the plane which has the maximum $f(h k l)$ value. In $\mathrm{CdO}$, the $f\left(\begin{array}{lll}1 & 1 & 1\end{array}\right)$ has the maximum value and hence its orientation is along that plane. Discussion on the manuscript is made only based on this assumption.

3. The change in $f$ factor may be due to the change in crystal structure and not to "reorientation effect".

- No, I cannot agree this argument. If for any particular concentration of $\mathrm{Zn}$ added to $\mathrm{CdO}$, if $f(1$ 11 ) has lesser value than $f\left(\begin{array}{lll}2 & 0 & 0\end{array}\right)$ then crystal starts to grow along that direction which is only due to 
reorientation effect. So it is not correct to say that $\mathrm{CdO}$ changes its structure at that $\mathrm{Zn}$ concentration.

4. The crystallite size in Table 2 should be presented without decimal digits. The systematic errors for evaluation of crystallite size from Scherrer formula are so high that so precise values are out of scientific meaning. Moreover, the Scherrer formula should NOT be used for quantitative crystallite size analysis. Moreover, the use of Scherrer method is not correct (although popular) due to several parameters of such calculations which should be taken into account, e.g, type of correction for instrumental broadening of diffraction lines, type of profile fitting, etc. [2, 3].
Without such information, the obtained values are of limited meaning.

- Yes, I accept that crystallite size values should be presented without decimal digits. But it is not a major error, as many reviewers in most journals accept crystallite size values with single or two decimal digits accuracy. I also agree that the Scherrer formula is not very accurate and crystallite size values calculated based on WilliamsonHall plots may be accurate. Also, corrections for instrumental broadening of diffraction lines, type of profile fitting, etc, must also be taken into account to get more accurate crystallite size values. 